\title{
Educación contra la pobreza legal para la consecución de los $\mathrm{ODS}$
}

Education against legal poverty to achieve the SDGs

\author{
Marta Bueno Salinas \\ Profesora titular de Historia del Derecho \\ Departamento de Historia del Derecho, \\ Derecho Romano y Derecho Eclesiástico \\ del Estado \\ Secretaria Académica de l'Institut de \\ Desenvolupament Professional (IDP- \\ ICE) \\ Universidad de Barcelona (España) \\ E-mail: martabueno@ub.edu
}

Resumen: Los últimos años han visto proliferar distintos estudios empíricos sobre el acceso a la Justicia, bajo el impulso de las metas generadas a partir de los Objetivos de Desarrollo Sostenible. Los datos muestran cuán alejados estamos de conseguir hacer efectivo el derecho a una Justicia para todos, tanto en su sentido estricto (derecho a obtener una resolución judicial), como en un sentido amplio de acceso a los derechos. Los Estados garantizan dicho acceso asumiendo los costes de la llamada 'justicia gratuita', si bien ello es manifiestamente insuficiente, pues 
solo una mínima parte de las cuestiones jurídicas son fruto de una controversia y/o requerirán un pronunciamiento judicial. Una educación jurídica básica puede contribuir a reducir los supuestos de pobreza o analfabetismo legal, supuestos que actúan como factores de riesgo para la exclusión social.

Palabras clave: ODS, Justicia, Pobreza, Exclusión, Educación.

\begin{abstract}
The last few years have seen the proliferation of empirical studies on access to Justice, prompted by the goals identified under the Sustainable Development Objectives. The data show how far we are from achieving the right to Justice for all, both in its strict sense (the right to obtain a judicial decision), and in a broad sense of access to rights. The States guarantee such access by underwriting the costs of the so-called 'free justice', although this is manifestly insufficient, since only a small part of the legal issues is the result of a controversy and/or will require a judicial ruling. Basic legal education can help reduce the cases of poverty or legal illiteracy, which act as risk factors for social exclusion.
\end{abstract}

Keywords: SDG, Justice, Poverty, Exclusion, Education.

\title{
1. Una reflexión previa
}

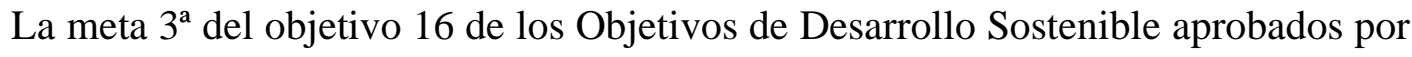
la ONU el 25 de septiembre del 2015 en el marco de la Agenda por el Desarrollo Sostenible se concreta en "promover el Estado de Derecho en los planos nacional e internacional y garantizar la igualdad de acceso a la justicia para todos." (ONU, 2015).

Esta doble meta aúna dos desiderátums de distinto alcance, aunque ambos están íntimamente relacionados: por un lado, la extensión de los Estados de Derecho como sistema sociopolítico y la consecuente aceptación de los principios que lo 
articulan como tal; por otro, el garantizar la igualdad de acceso a la justicia para todos, uno de los principios básicos y estructurales de todo Estado de Derecho.

En marzo de 2020 World Justice Project (entre otros organismos y entidades) propuso añadir un tercer indicador a los dos adoptados por la Asamblea general de la ONU en julio de 2017 para el seguimiento de la meta indicada, dado que aquellos indicadores atenían exclusivamente a aspectos relacionados con la justicia penal. (WORLD, 2020a). La propuesta, dirigida a reflejar "las necesidades cotidianas de justicia civil experimentadas por la mayoría de la población, especialmente los pobres, y los grupos que tienen el mayor riesgo de quedar rezagados", fue aceptada. El tercer indicador se ha concretado en medir la “[p]roporción de la población que se ha visto implicada en alguna controversia en los dos últimos años y ha accedido a algún mecanismo oficial u oficioso de solución de controversias, desglosada por tipo de mecanismo." " Se trata, en definitiva, de colocar "a las personas en el centro de los sistemas de justicia, y a la justicia en el centro del desarrollo sostenible." (PATHFINDERS, 2020: 17). ${ }^{2}$

Los datos de 2019 que proporciona el World Justice Project lo corroboran: sobre 7.700 millones de personas,

- $\quad 1.500$ millones de personas por año no obtienen solución a sus problemas jurídicos;

- $\quad 4.500$ millones de personas están excluidas de las oportunidades que prevé la ley;

- $\quad 235$ millones de personas viven en extremas condiciones de injusticia;

- $\quad$ Solo 1/3 de la población mundial obtiene una solución parcial (9\%) o total $(24 \%)$ a sus problemas jurídicos;

\footnotetext{
1 Vid. los indicadores respectos de los datos españoles en https://www.ine.es/dyngs/ODS/es/objetivo.htm?id=5252

2 Al consultar los datos del Informe en español téngase en cuenta que las cifras han sido mal traducidas desde el inglés.
} 
Solo entre el $5 \%$ y $6 \%$ de los asuntos se resuelven judicialmente. (WORLD, 2019).

Con datos parecidos, la organización HiiL user friendly justice estima que, de cada 8 millones de personas, 1 millón tendrá que enfrentarse anualmente a problemas de índole jurídica. (HIIL, 2018: 18).

Referidos a datos españoles, y no teniendo todavía los resultados oficiales del tercer indicador, de una muestra de 1051 ciudadanos, el 72\% afirmó haber tenido problemas jurídicos en los dos últimos años, en relación a temas variados: enfermedad y lesión derivadas de accidentes; ciudadanía e identificación; comunidad y recursos naturales; consumo; empleo; educación; familia; vivienda; tenencia tierra; cumplimiento de la Ley; dinero y deudas; servicios públicos. De este $72 \%$, solo un $39 \%$ manifestó haber podido acceder a ayuda para resolver el tema. Y de éstos, un escaso 7\% lo resolvió en instancias judiciales. (WORLD, 2019). ${ }^{3}$

Por otra parte, en los datos de World Justice Project 2020 para valorar los ítems que fundamentan el nivel de los Estados de Derecho, España mejoró su posición anterior (21), pasando a ocupar el puesto 19 de 128 países. De manera concreta al Factor 4, relativo al respeto de los derechos fundamentales, España ocupa la posición 16; mientras que en el Factor 7, sobre al acceso a la Justicia civil, desciende al número 25 debido, especialmente, a retrasos injustificados, fallos en la eficacia y puntualidad en la ejecución de las decisiones judiciales y a influencias políticas indebidas; por el contrario, en materia de Justicia penal, España se coloca en el número 20. (WORLD, 2020b)

Volvamos a los datos globales. 1.500 millones de personas no obtienen solución a sus problemas jurídicos. Pero, todavía es mayor la cifra de personas que, globalmente, están de entrada excluidas de las oportunidades legalmente previstas: 4.500 millones de personas. A estas dos cifras debemos sumar los 235 millones de personas que viven en situaciones de extrema injusticia. Unas y otras, por distintas

\footnotetext{
${ }^{3}$ Los datos se obtuvieron en 2018.
} 
razones, se encuentran objetivamente ante dificultades para acceder a los derechos, cuando no ante una total imposibilidad de acceso.

"La justicia es el hilo conductor que une los 17 Objetivos de Desarrollo Sostenible. Sin una mayor justicia, el mundo no podrá dar fin a la pobreza, reducir la desigualdad, asistir primero a los más excluidos, crear condiciones para oportunidades compartidas y sostenibles de prosperidad, ni tampoco promover la paz y la inclusión.” Así lo afirma y fundamenta la organización Pathfinders for Peaceful, Just and Inclusive Societies. (PATHFINDERS, 2020).

Casi más importantes que las cifras expuestas son las razones que subyacen. ¿Qué es lo que dificulta o imposibilita que la mayor parte de la población mundial no acceda al Derecho? Solo tras analizar dichas causas podrá actuarse y hacer efectiva la "Justicia para todos".

\section{El acceso al Derecho}

Las reflexiones sobre el acceso a los derechos suelen centrarse en cómo se facilita por los Estados el recurrir ante los tribunales, hoy en general extendido al asesoramiento previo. Indudablemente no es cuestión menor, en la medida en que no basta con tener reconocidos formalmente unos derechos, sino que es necesario poder ejercerlos materialmente, reservándose la instancia judicial para las reclamaciones derivadas de su violación o contravención. (MADRID, 2010: 34) El derecho fundamental a la tutela judicial efectiva (como proclama el artículo 24 de la Constitución española) es determinante para el acceso y la garantía de otros derechos. Por ello, nos encontramos ante un derecho en gran medida bisagra y de ahí su importancia. Pues, ¿cómo reclamar judicialmente la violación de un derecho (sea o no fundamental) si no podemos acceder a la Justicia? Ahora bien, el acceso a los tribunales es, en rigor, solo un aspecto de la categoría superior 'acceso a los derechos'.

\footnotetext{
${ }^{4}$ Vid., para distintas acepciones sobre 'acceso a los derechos': LIBRO BLANCO (2019: 5 y ss.).
} 
Los Estados sociales y democráticos de Derecho arbitran sistemas para intentar paliar las desigualdades, las barreras u obstáculos que dificultan o impiden el acceso a los derechos. ${ }^{5}$ En la consecución de este objetivo, Carrier-Plante et al. distinguen cinco distintos momentos a los que califican de 'olas' referidas al sistema norteamericano (CARRIER-PLANTE, 2016):

1. Década de 1960: se produce la democratización de la representación jurídica bajo la idea que debe garantizarse al acceso a los Tribunales. En esta década, y para hacer efectivo dicho acceso, se introducen los primeros programas gubernamentales de justicia gratuita; y, de manera paralela, con origen en USA, se empiezan a extender las llamadas clínicas jurídicas.

2. Década de 1970: se procede a la reforma de los sistemas judiciales, permitiendo el recurso de acciones colectivas, introducción de tribunales para asuntos de menor cuantía, tribunales administrativos especializados, etc. ${ }^{6}$

3. Decenio 1980-90: se apuesta por reforzar las capacidades de los justiciables vía la educación y la información jurídica, etc., así como se promueve la solución alternativa de los conflictos. ${ }^{7}$

4. Entre 1990 y 2000: la tendencia que se sigue busca empoderar a los ciudadanos vía la introducción de medios para evitar conflictos y una gradual concepción participativa del Derecho. ${ }^{8}$

5. En la fase actual asistimos a la búsqueda de representatividad del mundo jurídico, promoviendo la inclusión de las distintas categorías de los ciudadanos en los diferentes operadores jurídicos (políticos, funcionarios, notarios, abogados...). Y no solo eso, también se trabaja en la apuesta por la resolución de conflictos en línea (HIIL, 2016).

\footnotetext{
5 Sobre la valoración de la información que se proporciona desde las instancias gubernamentales sobre el acceso a los derechos vid., para España, WORLD (2020c).

${ }^{6}$ Buena descripción en PLANCHADELL (2015: 5-8).

${ }^{7}$ Mayor concreción en MANCHO (2014).

${ }^{8}$ Para relación con calidad democrática, vid. CASTELLLANOS (2019).
} 
Como ha quedado recogido, las barreras económicas han sido históricamente las primeras en ser eliminadas, al menos en lo que respecta al acceso a la Justicia, esto es a los Tribunales. En el entorno europeo, ello queda conceptualizado bajo el derecho a la asistencia jurídica gratuita, vinculada al acceso a los Tribunales. Este derecho aparece efectivamente con el modelo de Estado asistencial al reconocérsele entonces un carácter social puesto en relación con el acceso a los derechos civiles, económicos y culturales y frente al carácter caritativo que tenía el llamado históricamente 'beneficio de pobreza'.

En nuestro sistema jurídico, el artículo 24 de la Constitución española ya mencionado recoge como derecho fundamental el derecho a la tutela judicial efectiva, en otras palabras, el derecho a acceder a la Justicia, al establecer en su apartado primero que "Todas las personas tienen derecho a obtener la tutela efectiva de los jueces y tribunales en el ejercicio de sus derechos e intereses legítimos, sin que, en ningún caso, pueda producirse indefensión.” Ello se adecúa a lo preceptuado en la Carta de los derechos fundamentales de la Unión europea. En ella, en su título VI, dedicado a la Justicia, el artículo 47 formula el derecho a la tutela judicial efectiva y a un juez imparcial al señalar que: "Toda persona cuyos derechos y libertades garantizados por el Derecho de la Unión hayan sido violados tiene derecho a la tutela judicial efectiva respetando las condiciones establecidas en el presente artículo." Estas condiciones se enumeran seguidamente para concretarse en las siguientes:

"Toda persona tiene derecho a que su causa sea oída equitativa y públicamente y dentro de un plazo razonable por un juez independiente e imparcial, establecido previamente por la ley.

Toda persona podrá hacerse aconsejar, defender y representar.

\footnotetext{
9 Para un análisis detenido, vid. GÓMEZ (1982). Breve, pero ilustrativo, CARRIER-PLANTE (2016: 19 y ss.).
} 
Se prestará asistencia jurídica gratuita a quienes no dispongan de recursos suficientes siempre y cuando dicha asistencia sea necesaria para garantizar la efectividad del acceso a la justicia."10

Como se lee en el apartado $3^{\circ}$, la Carta europea no solo eleva a categoría de derecho fundamental el acceso a la justicia, sino que lo garantiza promoviendo su gratuidad para aquellos que no dispongan de recursos suficientes. También nuestra Constitución así lo prevé, pero no en sede de derecho fundamental, sino en su artículo 119, comprendido en el Título VI, dedicado al Poder judicial. En dicho artículo se prevé que "La justicia será gratuita cuando así lo disponga la ley y, en todo caso, respecto de quienes acrediten insuficiencia de recursos para litigar."11 No obstante, el análisis comparado de quiénes obtienen justicia gratuita refleja ciertas disfunciones en la medida en que ciudadanos con rentas medio-bajas, no susceptibles de ser beneficiarios de justicia gratuita, tampoco recurren a los servicios de abogados por el coste que representa. ${ }^{12}$ En países de nuestro entorno como Alemania, Bélgica, Escocia, Finlandia, Francia, entre otros- ello ha conducido a fomentar el contratar seguros de asesoramiento y defensa judicial, lo que ya empieza a introducirse tímidamente en nuestro país.

En cualquier caso, la reflexión y respuesta política europea actual sigue muy vinculada a la imperfecta sinonimia 'acceso a los derechos' = 'obtención de una resolución judicial'. ${ }^{13}$ Imperfecta sinonimia ya que no todos los problemas jurídicos se resuelven necesariamente accediendo a los tribunales. De hecho, es una parte considerablemente mínima aquella que sí culmina con una resolución judicial,

\footnotetext{
10 Esta misma proclama aparece en el artículo 19 del Tratado de la Unión cuando señala "Los Estados miembros establecerán las vías de recurso necesarias para garantizar la tutela judicial efectiva en los ámbitos cubiertos por el Derecho de la Unión”, en TRATADO (2012).

${ }^{11}$ Este mandato constitucional se concretó en la Ley 1/1996, de 10 de enero, de Asistencia Jurídica Gratuita. Disponible, con las sucesivas actualizaciones, en https://www.boe.es/buscar/act.php?id=BOE-A-1996-750

Vid. la propuesta de 2010 del Consejo de la Abogacía europea sobre el ámbito del acceso a la justicia, elevando a derecho fundamental la propia asistencia jurídica gratuita en CCBE (2010).

${ }^{12}$ Libro Blanco (2019: 31): "Our analysis of the WJP GPP data showed that in several countries, the lowest income group has significantly better access to assistance than the second lowest and middleincome groups."

${ }^{13}$ Vid.: http://ec.europa.eu/civiljustice/legal_aid/legal_aid_spa_es.htm
} 
como claramente muestra el gráfico de la Oficina del Fiscal General de Australia (LIBRO BLANCO, 2019: 6):

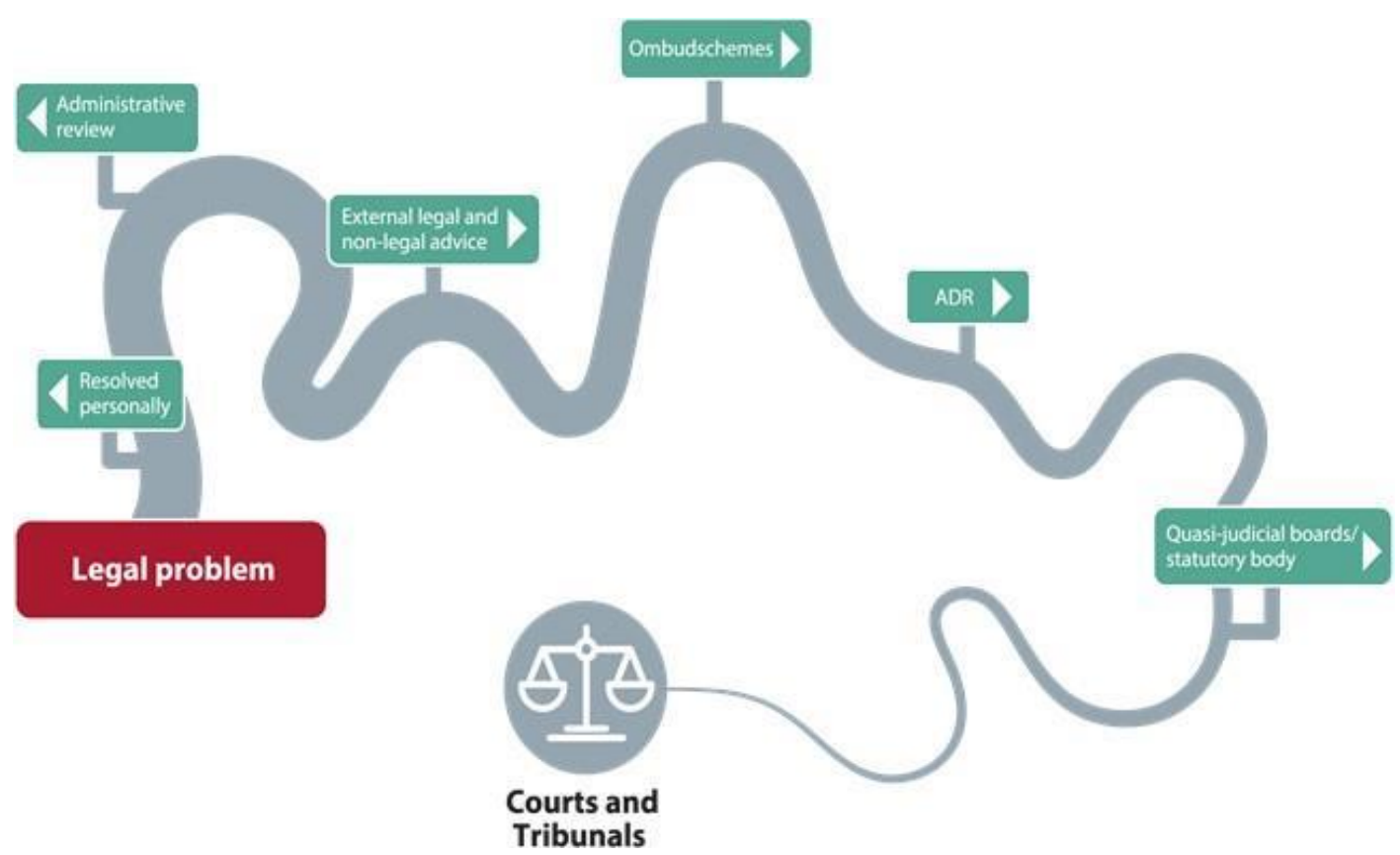

Las previsiones constitucionales aseguran el acceso a la Justicia, el derecho a la obtención de una sentencia judicial. ${ }^{14}$ Pero, como acabamos de ver, las dificultades jurídicas que asoman a la ciudadanía solo en pocas ocasiones consiguen -lo requieran o no- una resolución judicial.

Efectivamente, no toda problemática jurídica ha de provocar necesariamente una intervención judicial. Como resume el gráfico reproducido, las cuestiones jurídicas de una y otra índole se resuelven de mayor a menor frecuencia:

- de manera personal;

- $\quad$ con intervención de la Administración;

- $\quad$ vía asesoramiento externo;

- con intervención de un defensor (de consumo, bancario, del paciente...);

vía conciliación, mediación o arbitraje;

\footnotetext{
${ }^{14}$ Para datos comparados de los sistemas judiciales (2018, última edición al escribir estas líneas) en la Unión europea, vid. CEPEJ-STAT.
} 
- $\quad$ a través de juntas u órganos estatutarios;

- $\quad$ acudiendo a los tribunales.

Por otra parte, ¿qué ocurre con todas aquellas situaciones en las que la cuestión jurídica que se plantea no es sinónima de una problemática sino de acceso a derechos en sentido estricto? Acceder a la sanidad, a la educación, a una ayuda social, no sufrir discriminación, entender la dimensión jurídica de una determinada situación, son solo algunos de los muchos ejemplos posibles. Aparentemente los textos constitucionales solo abordan el acceso a una resolución judicial como paradigma del acceso a los derechos. Pero, son muchas las situaciones que no requieren de un pronunciamiento judicial y/o ni siquiera descansan en una controversia jurídica. ¿Cómo podemos afirmar que somos ciudadanos con derechos si el acceso a los mismos no está garantizado? Son los retos del acceso a la justicia de los que habla LAMARCHE (2016).

\section{La pobreza o analfabetismo legal}

Las dificultades de acceso a los derechos no son -como veremos- de naturaleza estrictamente jurídica sino también social o personal: niveles bajos de alfabetización, falta de conocimientos e información, obstáculos lingüísticos, hándicaps psíquicos o intelectuales, discriminación racial, falta de recursos financieros, ${ }^{15}$ son solo algunas de las causas, a las que podemos añadir la distancia geográfica de los núcleos urbanos o no disponer de internet. En este sentido, y a modo de ejemplo, el Colegio de Abogados canadiense concluyó en un estudio del 2013 que un $48 \%$ de los ciudadanos canadienses no disponían de las competencias necesarias para beneficiarse de la información jurídica accesible en línea o de otra manera. ${ }^{16}$

Dificultar, en el sentido de no facilitar, o incluso imposibilitar, el acceso a los derechos ahonda la desigualdad, los riesgos de exclusión social de los colectivos

15 McLACHLIN (2016: 344). El análisis reflexiona especialmente en torno a los casos que llegan efectivamente a los tribunales, pero no contempla qué ocurre con los que ni siquiera llegan. Vid. también BAHARY-DIONNE (2018: 341 y ss.).

16 Dato tomado de LAMARCHE (2016: 25), quién añade: "Difficile, donc, d'imaginer que ces citoyennes et ces citoyens constituent des utilisateurs déraisonnables du système de justice." 
más vulnerables. Pero ¿solo de ellos? El no acceso al Derecho, especialmente en momentos de máxima judicialización como la actual, puede provocar que colectivos que a priori parecerían estar alejados de ser calificables de estar en riesgo de exclusión social, acaben conformando la que consideramos una nueva categoría sociológica: la pobreza legal.

El concepto de 'pobreza legal', no empleado por el momento en la literatura académica española, se debe a Fernando del Mastro Puccio, profesor en la Facultad de Derecho de la Pontificia Universidad Católica de Perú, quien publicó en 2007 un artículo bajo el título "Pobreza legal y pobreza legal extrema: ¿quiénes son los responsables?" (DEL MASTRO, 2007).

Del Mastro Puccio refiere bajo el concepto 'pobreza legal' a "la incapacidad de una persona para poder utilizar las normas, las instituciones del Estado y otros mecanismos alternativos como medio idóneo para poder ejercer derechos y solucionar conflictos." (DEL MASTRO, 2007: 260). Desde un punto de vista subjetivo, con la vista puesta en la incapacidad de la persona a que refiere Del Mastro Puccio, esta incapacidad puede venir dada por barreras idiomáticas (por ejemplo, desconocer el único idioma usado por parte de la Administración), geográficas (como vivir muy alejado de las instancias administrativas o judiciales), económicas (no disponer de recursos para iniciar un procedimiento administrativo o judicial) o de propia comprensión de la temática jurídica, dada el lenguaje propio que emplea el Derecho y el elevado nivel conceptual sobre el que se construye, haciendo necesaria la intervención de un especialista. El que se encuentra en situación de 'pobre legal' conoce sus derechos, pero factores externos a él le imposibilitan acceder a los mismos.

En este mismo orden de ideas, pero desde un punto de vista objetivo, Madrid Pérez distingue cuatro grupos de causas, entrelazables, que obstaculizan -pudiendo llegar a impedir- el acceso a los derechos: causas económicas, sociales, culturales e institucionales. (MADRID, 2010: 41 y ss.). Todas ellas, cuanto más se alejan de los estándares mínimos, más inciden negativamente en la necesaria conciencia jurídica que requiere el efectivo acceso a los derechos. Porque, ciertamente, "[u]na cultura 
legal débil, tanto por parte de las autoridades como por parte de la ciudadanía, contribuye a incrementar la exclusión social y a limitar el acceso a los derechos." (MADRID, 2010: 43). ${ }^{17}$

Madrid Pérez hace referencia -como hemos visto- a una 'cultural legal débil' para referir a aquellas situaciones en las que, conociendo sus derechos, factores externos impiden su ejercicio. Esta descripción coincide con el concepto 'pobreza legal'. Pero, Del Mastro Puccio da un paso más al diferenciar entre la 'pobreza legal' y la 'pobreza legal extrema'. Ésta refiere a “aquella situación de desconocimiento total de derechos y/o de mecanismos básicos previstos en el Estado para solucionar conflictos o ejercer derechos." (DEL MASTRO, 2007: 260). Estas situaciones se califican también de 'analfabetismo legal' o de falta de capacidad legal, ${ }^{18}$ no teniendo por qué estar relacionadas con circunstancias económicas adversas, circunstancias geográficas, familiares, sociales o circunstancias derivadas del llamado 'analfabetismo funcional', esto es -según definición de la UNESCO, 2006la incapacidad de realizar actividades en las que es necesaria la alfabetización para la actuación eficaz en el grupo o comunidad. La 'pobreza legal extrema' identifica una situación que descansa en la ignorancia. Y es evidente que desconocer la existencia de un derecho impide el acceso al mismo.

El desconocimiento en torno a la existencia de unos derechos que son exigibles en los Estados puede afectar directamente al ciudadano o, incluso, a las propias autoridades encargadas de la prestación de servicios públicos. En este sentido, cabe pensar, por ejemplo, en situaciones en las que se ignore por parte del trabajador en una Administración la existencia de concretas ayudas o desconocer situaciones de gran vulnerabilidad por razón de edad y soledad en sociedades altamente envejecidas como la nuestra. Piénsese, por ejemplo, el siguiente supuesto real: dos hermanas octogenarias comparten vivienda. Son autónomas e independientes. Una

17 Vid., en este mismo sentido, las interesantes observaciones de la Abogacía canadiense (ASSOCIATION, 2013).

18 CASTILLA (2012: 35 y ss.) y LIBRO BLANCO (2019: 25) califican de 'capacidad legal' el reconocer problemas jurídicos, saber moverse por los servicios y procedimientos judiciales y saber resolver problemas legales. Según los datos que proporciona, un 20\% de las personas encuestadas en los distintos países analizados adolecerían de falta de capacidad legal. 
de ellas, la mayor, es la que lleva el día a día de la casa hasta que sufre un ictus que la deja casi postrada en la cama, perdiendo la capacidad de comunicación oral y escrita. La hermana pequeña asume las funciones de la mayor, así como su cuidado. La situación se va deteriorando día a día, sin que llegue a conocimiento de los servicios sociales. Este supuesto encajaría en el de 'pobreza legal' ante la dificultad de acceder a todos aquellos derechos previstos para situaciones como la descrita: posibilidad de acceder a los servicios sociales, de nombrar un tutor legal, de tramitar ayudas...

Los sujetos en situación de pobreza y/o de analfabetismo legal, sea o no extrema dicha pobreza, acaban por desdibujarse, por pasar inadvertidos, por hacerse invisibles para el Estado. Según ya hemos comentado, 4.500 millones de personas se encuentran en esa situación. Y a esta cifra deberíamos sumar los 235 millones de personas que viven en situaciones de extrema injusticia.

\section{Medidas contra la pobreza o el analfabetismo legal}

Más allá de la previsión constitucional, más allá de las concretas políticas públicas tendentes a satisfacer los derechos reflejos de las necesidades sociales, la sociedad civil ha desarrollado prácticas dirigidas a facilitar el acceso a los derechos a toda la ciudadanía. En este sentido, entidades o asociaciones sin ánimo de lucro, del llamado 'tercer sector', asumen funciones de asesoría jurídica, representación gratuita ante los Tribunales, de formación a sectores vulnerables o personas en exclusión, llegando a un mayor número de personas de las susceptibles de ser beneficiadas por la actividad directa de la Administración. ${ }^{19}$

Por otra parte, desde las Universidades se articulan también programas dirigidos a facilitar el acceso a los derechos a los colectivos a priori más vulnerables. Son las llamadas 'clínicas jurídicas'. Éstas fueron introducidas en Estados Unidos a partir

\footnotetext{
${ }^{19}$ Vid., por ejemplo, para datos del área metropolitana de Barcelona, BARÒMETRE (2020).

El Consejo General de la Abogacía Española publica anualmente, desde el año 2006, el Informe del Observatorio de Justicia gratuita. (CONSEJO).

Las cifras son también recogidas en WORLD (2019), portal que permite la comparación con otros países.
} 
de la reflexión de Jerome N. Frank, quien publicó en 1933 el artículo "Why not a clinical lawyer-school?" (FRANK, 1933).

Las clínicas jurídicas responden -como sintetiza MADRID (2010: 49) explicando el proyecto dret al Dret de la Facultad de Derecho de la Universidad de Barcelona- "a una idea clave para una sociedad democrática que se organiza institucional y jurídicamente como un Estado de Derecho: garantizar el acceso efectivo a los recursos jurídico-sociales que favorecen el ejercicio de los derechos." A ello se suman otras dos preocupaciones: "cómo mejorar el aprendizaje y la formación jurídica de los estudiantes y cómo potenciar el servicio público que ha de prestar la Universidad.",20

Últimamente también se está generalizando la actividad 'pro bono' por parte de los despachos profesionales, en atención a la llamada 'responsabilidad social corporativa'. (SANTOS, 2018). La actuación pro bono supone la colaboración de un despacho o empresa profesional con una entidad del tercer sector, ofreciendo aquélla a ésta el trabajo gratuito de uno de sus profesionales para atender proyectos o casos concretos, cuando no asumiendo el asesoramiento y/o representación directa de un determinado ciudadano en atención al interés global que el tema pueda suscitar. No debe confundirse con una colaboración de voluntariado porque la participación del profesional se realiza bajo horario laboral, asumiendo el despacho o empresa el coste económico de dicha colaboración. ${ }^{21}$

En algunos países se abren otras posibilidades, como la experiencia del Estado de Nueva York o de Washington: se prepara con unas mínimas nociones jurídicas a ciertos ciudadanos (los llamados 'parajuristas') con la idea de que asesoren a personas en situación de vulnerabilidad, asistiéndolas en determinados tipos de reclamaciones judiciales. (McLACHLIN, 2016: 346).

20 Vid., también, la concreción de las distintas clínicas del dret al Dret en https://www.ub.edu/portal/web/dret/cliniques-dret-al-dret

${ }^{21}$ Para las diferencias, vid. WORK (2018). 
En cualquier caso, todas estas experiencias han de superar el recelo que generan en el conjunto de la Abogacía. ${ }^{22}$ Solo recientemente se ha dado algún paso en la colaboración entre las clínicas jurídicas y los colegios de abogados, como muestra el todavía proyecto de Convenio entre la Conferencia de Rectores de las Universidades y el Consejo General de la Abogacía. (CRUE, 2018).

Por su parte, también las Administraciones introducen medidas para paliar las barreras u obstáculos que dificultan el acceso a los derechos. En este sentido, y más allá de ventanillas destinadas al asesoramiento en general, las Administraciones tienden a hacerse eco de la diversidad y pluralidad de origen de sus ciudadanos e intentan facilitar la información en diversos idiomas, pudiendo incluso determinar la participación de traductores en aquellas áreas consideradas más sensible, como puede ser el área de sanidad. De la misma manera se busca hacer desaparecer las barreras geográficas aprovechando la extensión del uso de internet colgando la información en los portales informáticos y facilitando de manera electrónica los pertinentes formularios, así como permitiendo su presentación telemática. Ahora bien, la indudable ventaja que encierra la extensión del uso de las redes telemáticas por parte de la Administración es inversamente proporcional a la edad de la población usuaria. Situaciones vividas bajo la reciente pandemia de la COVID19, con la prestación de servicios totalmente en línea y con el consiguiente cierre de ventanillas, pueden dificultar el acceso a los derechos. ${ }^{23}$

En definitiva, si bien el legislador acepta la previsión legal de garantizar el acceso a los tribunales como indiscutible derecho vinculado al acceso a los derechos, dicha previsión se queda corta en su materialización porque no solo es una cuestión de recursos económicos la que puede dificultar o llegar a impedir el acceso a los

\footnotetext{
${ }^{22}$ Vid. INFORME (2020: 191), sugerencia 20, muy cauto respecto de la actuación pro bono.

23 JIMENEZ (2020): "La brecha digital, por mucho que se ignore (también por la Administración), sigue siendo algo real y doloroso. Y no se sabe a ciencia cierta cuántas personas se han quedado fuera de esos circuitos de ayudas al no poder informarse o tramitarlos por cauces telemáticos (por ejemplo, no deja de ser curioso que los locutorios hayan sido uno de los servicios más demandados en esta crisis por tales colectivos). La ética de orientación al servicio de la ciudadanía como premisa de actuación de la Administración Pública en parte ha quebrado durante esta primera fase de la pandemia."

Sobre la protección de los derechos humanos en pandemia vid. SAUQUILLO (2021).
} 
derechos. Por ello, creemos que el Estado debería garantizar no solo el acceso a los tribunales sino en general el acceso a los derechos con políticas activas en distintos órdenes, especialmente en materia de educación, porque ¿cómo reclamar si es posible que ni siquiera se conozca la existencia de un derecho?

\section{La mejor apuesta, la educación: el empoderamiento o alfabetización jurídica}

El acceso a los derechos requiere estar informado sobre qué significa 'tener derecho'. Y esa información solo es posible tenerla si se está educado en ello. (ASSOCIATION, 2013: 18 y ss.). En este sentido, es muy interesante la iniciativa llevada a cabo en 2009 por el Observatorio de la Exclusión Social y los Procesos de Inclusión en la Comunidad de Madrid: De la exclusión a la ciudadanía. Análisis de los factores, barreras y oportunidades (EISM, 2009). La elaboración del informe contó con la participación de personas en situación de exclusión quienes, en distintos talleres, trabajaron diferentes temas relacionados con su percepción sobre el 'acceso a los derechos'. Este tipo de estudios empíricos, todavía muy escasos en España, son necesarios para poder perfilar respuestas político-sociales más ajustadas. ${ }^{24}$ Las conclusiones obtenidas remarcan, para reforzar los vínculos y canales de acceso a los derechos de los colectivos vulnerables, la necesidad de "profundizar en el conocimiento de los derechos que tienen los participantes de nuestros proyectos de inserción, así como facilitar información-formación de los recursos de los que disponen para poder acceder a ellos (justicia gratuita, ventanillas administrativas, oficinas de atención ciudadana, órganos de participación y consulta, etc.).” Pero, no ha de acabarse ahí. Debe incidirse de manera proactiva en aquellas situaciones de vulnerabilidad que, descansando en causas calificables de pobreza legal, pueden conducir a la exclusión.

Apostar por una mínima educación legal no ha de suponer vulgarizar (en el sentido de popularizar) lo jurídico provocando la pérdida de la especialidad que

\footnotetext{
${ }^{24}$ Vid. valoración concreta sobre cómo deben ser los estudios empíricos relacionados con 'educar en lo legal' en WILCZYNSKI (2014).
} 
comporta. ${ }^{25}$ Sí significa formar a ciudadanos para que sean conscientes de sus derechos y deberes, así como mínimamente conocedores de lo jurídico. En los últimos años, y con el objetivo de aproximar el Derecho al conjunto de la ciudadanía, están proliferando websites o plataformas en línea en las que se explican temas jurídicos básicos en orden a hacerlos accesibles. Otras actuaciones apuestan por la formación directa. En este sentido, la República Checa, en posición 18 en la relación países/Estado de Derecho, (14 en Derechos fundamentales, 20 en Justicia civil (por retrasos, ejecución y accesibilidad) y 16 en Justicia penal), introdujo una asignatura obligatoria de Derecho en los estudios universitarios, así como en los centros de secundaria, apostando por la alfabetización jurídica con proyectos diversos. (KRUPOVÁ 2017). Además de clínicas jurídicas, impulsan el llamado 'Street Law', esto es, la participación de estudiantes de Derecho en la formación jurídica de los ciudadanos en general. Estas experiencias son parejas a las que se engloban bajo la denominación 'Law in Action', con buenos ejemplos en USA y Canadá. ${ }^{26}$

Indiscutiblemente, educar en lo jurídico revierte en positivo al conjunto de la sociedad y sus instituciones. (LIBRO BLANCO, 2019: 45). Así lo entienden instituciones como The Legal Education Fundation, muy activa en Reino Unido: "We focus on the essential role of legal education in helping people and organisations to understand and use the law as a tool for change. In this work, we know that there are significant numbers of people who cannot obtain justice in relation to everyday problems, and that these unmet needs trigger or exacerbate clusters of issues that have a social and economic impact on individuals, communities and wider public policy goals. We believe the law should be readily available at the times and in the places where people need it." 27

\footnotetext{
25 De hecho, una de las razones consideradas clave que explican la falta de 'capacidad legal' para determinados tipos de problemas es la especialización o argot jurídico. Vid. LIBRO BLANCO (2019: 27). En cualquier caso, menos del 25\% de los ciudadanos encuestados dicen resolver sus temas jurídicos con asesoramiento de un abogado, intervención de una oficina de asistencia jurídica, o de un órgano judicial o administrativo.

${ }^{26}$ Vid., por ejemplo, https://law.wisc.edu/law-in-action/, https://www.lawinaction.ca/.

${ }^{27}$ https://www.thelegaleducationfoundation.org/
} 
Efectivamente, "[1]a educación es el principal medio que permite a adultos y menores marginados económica y socialmente salir de la pobreza y participar plenamente en sus comunidades." De manera rotunda, lo afirma la introducción de la Observación General 13, dictada en 1999 por el Comité de Derechos Económicos, Sociales y Culturales del Consejo Económico y Social de las Naciones Unidas sobre el derecho a la educación. (COMITÉ 1999).

En esta misma línea, el objetivo 4 de los ODS de la Agenda 2030 apuesta expresamente por "garantizar una educación inclusiva, equitativa y de calidad y promover oportunidades de aprendizaje durante toda la vida para todos." Y significativamente, su meta $7^{\mathrm{a}}$ reconoce la necesidad de educar en derechos humanos (esto es, los derechos fundamentales) al señalar que debe asegurarse "que todos los alumnos adquieran los conocimientos teóricos y prácticos necesarios para promover el desarrollo sostenible, entre otras cosas mediante la educación para el desarrollo sostenible y los estilos de vida sostenibles, los derechos humanos, la igualdad de género, la promoción de una cultura de paz y no violencia, la ciudadanía mundial y la valoración de la diversidad cultural y la contribución de la cultura al desarrollo sostenible." ${ }^{28}$ En definitiva, debe educarse y concienciar a la ciudadanía no solo de la existencia de unos derechos mínimos, sino también en relación a su exigibilidad.

Las sociedades democráticas requieren ciudadanos educados en valores compartidos. (BUENO, 2019). Entre estos valores se encuentra conocer la existencia de derechos estimados básicos. Solo una educación en ese sentido, una educación que promueva y de esta manera garantice la posibilidad de acceso a los derechos, puede contribuir efectivamente a que la calificada de 'pobreza legal' desaparezca como factor de riesgo de exclusión. Así lo entendió la Asociación de abogados canadienses, lo que le llevó a apoyar, sin reserva, la creación de organizaciones no gubernamentales en cada provincia con la misión de formar e informar a la ciudadanía sobre qué significa tener derechos y sobre el propio

${ }^{28}$ ONU (2015) y OEISM (2012).

Para un recorrido en relación al derecho a la educación en un mundo globalizado, vid. MARTÍNEZ (2014: 206 y ss.). 
sistema jurídico. ${ }^{29} \mathrm{Y}$ es que el acceso a los derechos deviene un factor clave en la generación o superación de desigualdades, pudiendo llegar a ser determinante para concluir la integración o no en el grupo social de referencia.

Y, ¿cómo plantearse dicha educación? ¿Qué mecanismos pueden contribuir a formar ciudadanos conscientes de sus derechos y obligaciones? Una mínima formación jurídica en todos los niveles educativos sería deseable. ${ }^{30}$ Mientras este objetivo se alcanza, pueden abrirse otras vías con el objetivo de aproximar el Derecho a la ciudadanía en general y a los representantes políticos en particular, puesto que muchos de ellos acceden a cargos de representación sin conocer unas reglas mínimas de lo que significa un Estado democrático y de Derecho y/o de conceptos jurídicos básicos.

Desde la Universidad estas otras vías deben impulsarse. En este sentido, dret al Dret promueve, participa y coordina en la Facultad de Derecho de la Universidad de Barcelona el proyecto 'Compartir ideas' del grupo ApS de la propia Universidad. ${ }^{31}$ Desde dret al Dret ofrecemos distintos temas relacionados con las diferentes titulaciones de Grado de la Facultad para ser explicados por nuestros estudiantes a estudiantes de Secundaria o Bachillerato. ${ }^{32}$ De esta manera, conseguimos acerca el Derecho a la enseñanza secundaria, ante la falta de contenidos curriculares relacionados con esta materia. Es una experiencia del llamado 'Street Law'.

Y desde el curso 2019-20, dret al Dret diseña y coordina la oferta de la Facultad de Derecho en la Universidad de la Experiencia de la Universidad de Barcelona con el programa de dos años 'Derecho y Estado social I y II', dirigido a mayores de 55 años, con o sin titulación universitaria previa. ${ }^{33}$ Otro ejemplo de 'Street Law'.

\footnotetext{
29 Vid. https://www.cba.org/For-The-Public/Public-Legal-Education-and-Information-in-Canada y las previas conclusiones del COMITÉ (2013).

${ }^{30}$ Vid. BUENO (2019) y las conclusiones de la nota inmediatamente anterior.

${ }^{31}$ http://www.ub.edu/grupapsub/es/article/nou-catàleg-compartir-idees-edició-2020

32 Además de Derecho y de diferentes dobles Grados, la Facultad ofrece los Grados de Ciencias Políticas y de la Administración, Criminología, Gestión y Administración Pública y Relaciones Laborales.

${ }^{33}$ http://www.ub.edu/experiencia/programes.php.
} 
Uno y otro programa son solo una pequeña muestra de lo que puede impulsarse desde la Universidad. Introducir líneas educativas en lo jurídico de manera generalizada sería no solo deseable, sino también conveniente. En definitiva, se trata de empoderar jurídicamente a la ciudadanía. Al formar a las personas, "saben actuar cuando surge una necesidad legal. Se les ayuda para comprender la ley y buscar una solución, mientras que se da asistencia jurídica a los más vulnerables." (PATHFINDERS, 2020: 21 y 74 y ss.). Es no solo la primera etapa hacia la consolidación de la Justicia sino, seguramente, la más relevante.

\section{A modo de síntesis}

La exclusión social va más allá de una situación provocada por razones reconducibles a la categoría 'pobreza económica'. Es resultado de un proceso en el que se concatenan diferentes déficits en una misma persona, potenciándose los unos con los otros. Esta múltiple dimensión determina que también deban ser múltiples las herramientas para eliminar los factores provocadores de desigualdad y conducentes a la exclusión. Los Estados sociales arbitran vía sus políticas públicas mecanismos para paliar las causas de vulnerabilidad. La triple dimensión de la misma (estructural o económica, contextual o social y subjetiva o personal) lleva a la necesidad de actuar en cada una de estas dimensiones en aras de evitar la exclusión social.

La aproximación al tratamiento doctrinal de la exclusión social, de sus causas o factores de riesgo, desvela la ausencia de referencias a un factor referido al acceso a los derechos: la pobreza o analfabetismo legal. La delimitación de este factor tiene en cuenta el reconocimiento en cuanto ciudadanos de la existencia de derechos fundamentales, esto es, derechos humanos positivizados. No poder ejercer dichos derechos por causas ajenas a la voluntad de sus titulares o desconocer la existencia de los mismos no solo impide su efectivo ejercicio, sino que, también, ahonda la vulnerabilidad que puede conducir a la exclusión social.

El propio derecho a la Justicia o de acceso a los Tribunales, es decir, a obtener una resolución judicial, queda garantizado en los ordenamientos de los Estados 
democráticos como derecho fundamental. Su protección se delimita determinando, además, supuestos en los que el acceso deviene por Ley gratuito. Pero, ello no es suficiente: hay casos en los que determinados ciudadanos no consiguen reclamar sus derechos bien por desconocimiento de los mismos o bien por desconocimiento de los mecanismos por los que pueden reclamarlos. Son los supuestos de pobreza o analfabetismo legal.

De manera paralela al funcionamiento del derecho a la asistencia jurídica gratuita, las sociedades demócratas occidentales van desarrollando otros mecanismos para paliar el riesgo de exclusión o la vulnerabilidad que supone la dificultad de acceder a los derechos o, incluso, ignorar la existencia de los mismos. En este sentido, son ilustrativos los servicios jurídicos que ofrecen entidades del Tercer Sector, las Clínicas jurídicas o la actuación pro bono de los despachos jurídicos profesionales. En cualquier caso, son medidas que, si bien pueden contribuir a paliar este factor de exclusión, no consiguen eliminarlo. Se requiere una política educativa de calado. Solo educando en valores democráticos, dotando de unos mínimos conocimientos jurídicos, formando en competencias/habilidades jurídicas, podremos conseguir que se tome conciencia de que son estos derechos los que hacen que no seamos meros individuos sino ciudadanos, esto es, sujetos titulares de derechos y obligaciones.

Zanjar el analfabetismo legal no va a acabar con la exclusión social en abstracto ni en general, pero puede hacer que una parte de ciudadanos en riesgo de exclusión no acaben excluidos; y puede conseguir a su vez que algunos ya en esa situación tengan la opción de reintegrarse socialmente reclamando sus derechos.

\section{Referencias bibliográficas}

ASSOCIATION (2013): Association du Barreau Canadien, Attendre l'egalité devant la justice: une invitation à l'imagination et à l'action. Disponible en https://www.cba.org/CBAMediaLibrary/cba_na/images/Equal\%20Justice\%20\%20Microsite/PDFs/Equal-Justice-Summary-Report-fra.pdf 
BAHARY-DIONNE (2018): Bahary-Dionne, Alexandra, "L'accès à la justice en contexte numérique: l'information juridique par et pour les justiciables sur les médias sociaux", en Windsor Yearbook of Access to Justice/Recueil annuel d'Windsor d'accès à la justice 35, 337-362. Disponible en https://www.erudit.org/en/journals/wyaj/2018-v35-wyaj04384/1061345ar/

BARÒMETRE (2020): Baròmetre del tercer sector social, edició 2020. Disponible en https://xarxanet.org/editorial-document/taula-dentitats-del-tercer-sector-socialde-catalunya-area-metropolitana-de

BUENO (2019): Bueno Salinas, Marta, "Educar para la Democracia", en Revista Educación $\quad y \quad$ Derecho $20 . \quad$ Disponible en https://revistes.ub.edu/index.php/RED/article/view/29695/30023

CARRIER-PLANTE (2016): Carrier-Plante, Charles/Duchesne Blondin, Alexandre/Fontan, Jean-Marc/Laniel, Richard-Alexandre/Sénéchal, Yan/Vaillancourt, Kevin, “L'accès à la justice : perspectives sociologiques", en L'accès à la justice, quelle justice?, Nouveaux Cahiers du socialisme 16, 15-21. Disponible en https://www.erudit.org/en/journals/ncs/2016-n16$\underline{\text { ncs02593/82645ac.pdf }}$

CASTELlanOS (2019); Castellanos Claramunt, J., "Empoderamiento y participación local: bases para un papel activo del ciudadano en la mejora de la democracia”, en Estudios de Gobierno Abierto, Diputación de Valencia, 1-19. Disponible en: https:/www.dival.es/sites/default/files/portal-detransparencia/03\%20Castellanos\%20Jorge.pdf

CASTILLA (2012): Castilla Juárez, Karlos Artemio, Acceso efectivo a la Justicia. Elementos y caracterización, Editorial Porrúa, México.

CCBE (2010): Consejo de la Abogacía europea, Recomendaciones de CCBE sobre Asistencia jurídica gratuita. Disponible en https://www.abogacia.es/wpcontent/uploads/2016/01/Recomendaciones-de-CCBE-sobre-Asistencia-juridicagratuita.pdf 
CEPEJ-STAT: European Commission for the Efficiency of Justice, Dynamic Database of European judicial systems, https://www.coe.int/en/web/cepej/dynamic-database-of-european-judicial-systems

COMITÉ (1999): Comité de Derechos económicos, sociales y culturales, Aplicación del Pacto Internacional de Derechos económicos, sociales y culturales. Observaciones generales 13. El derecho a la educación. Disponible en https://www.right-to-education.org/sites/right-to-education.org/files/resourceattachments/ONU_Observación_General_13_Derecho_Educación_es.pdf

COMITÉ (2013): Comité d'action sur l'accès á la justice en matière civile et familiale, L'accès a la Justice en matière civile et familiale. Une feuille de route pour le changement. Disponible en https://www.cfcjfcjc.org/sites/default/files/docs/2013/AC_Report_French_Final.pdf

CONSEJO (desde 2006), Consejo general de la Abogacía, Informes del Observatorio de Justicia gratuita https://www.abogacia.es/publicaciones/abogaciaen-datos/observatorio-de-justicia-gratuita/

CRUE (2018): Convenio Colaboración CGAE-CRUE. Disponible en https://www.academia.edu/37619229/Borrador_Convenio_Colaboracio_n_CGAE_ CRUE_Limpio_docx

DEL MASTRO (2007): Del Mastro Puccio, Fernando, "Pobreza legal y pobreza legal extrema: ¿quiénes son los responsables?”, en Themis. Revista de Derecho 53, 255-266.

Disponible http://revistas.pucp.edu.pe/index.php/themis/article/view/8861

FRANK (1933): Frank, Jerome, "Why not a clinical lawyer-school?", https://digitalcommons.law.yale.edu/cgi/viewcontent.cgi?referer=https://www.goog le.com/\&httpsredir=1\&article=5092\&context=fss_papers

GÓMEZ (1982): Gómez Colomer, Juan-Luis, El beneficio de la pobreza: la solución española al problema del acceso gratuito a la justicia, Barcelona. 
HIIL (2016): HiiL user friendly justice, ODR and the Courts: The promise of 100\% Access to Justice. Disponible en https://www.hiil.org/wpcontent/uploads/2018/09/Online-Dispute-Resolution-Trend-Report.pdf

HIIL (2018): HiiL user friendly justice, Understanding Justice Needs: The Elephant in the Courtroom. Disponible en https://www.hiil.org/projects/understanding-justice-needs-the-elephant-in-thecourtroom/

INFORME (2020), Informe XIV del Observatorio de Justicia Gratuita. Disponible en https://www.abogacia.es/wp-content/uploads/2020/07/XIV-Informe-delObservatorio-de-Justicia-Gratuita-def.pdf

JIMENEZ (2020): Jiménez Asensio, Rafael, "Pandemia, vulnerabilidad social y Administración pública”. Disponible en https://hayderecho.expansion.com/2020/05/25/pandemia-vulnerabilidad-social-y$\underline{\text { administracion-publica/ }}$

KRUPOVÁ (2017): Krupová, T./Zima, M. (2017), "Street Law and Legal Clinics as Civic Projects: Situation in the Czech Republic", Oñati Socio-Legal Series, vol. 7, nº 8. Disponible en http://opo.iisj.net/index.php/osls/article/view/775

LAMARCHE (2016): Lamarche, Lucie, "Les enjeux de l'accès à la justice à l'heure de la philanthropie, de l'empowerment et de l'austérité: illusions et confusión", en L'accès à la justice, quelle justice? Nouveayu Cahiers du Socialisme 16, 22-33. Disponible en https://www.erudit.org/en/journals/ncs/2016$\underline{\mathrm{n} 16-\mathrm{ncs} 02593 / 82646 \mathrm{ac} /}$

Ley 1/1996, de 10 de enero, de Asistencia Jurídica Gratuita. Disponible, con las sucesivas actualizaciones, en https://www.boe.es/buscar/act.php?id=BOE-A-1996$\underline{750}$ 
LIBRO BLANCO (2019): OCDE y World Justice Project, Building a Business Case for Access to Justice. Disponible en https://www.oecd.org/gov/building-abusiness-case-for-access-to-justice.pdf

MADRID (2010): Madrid Pérez, Antonio: "El acceso a los derechos: la experiencia del Proyecto dret al Dret”, en Anuario de Filosofía del Derecho 26, 31-56.

MANCHO (2014): Mancho Gómez, Carolina, "Origen y evolución de la mediación: el nacimiento del «movimiento ADR» en Estados Unidos y su expansión a Europa”, en Anuario de Derecho Civil 67.3, 931-996.

MARTÍNEZ (2014): Martínez de Pisón, José, "Derechos sociales y cohesión global: una educación para todos”, en Ma José Bernuz Benéitez/Manuel Calvo García, La eficacia de los derechos sociales, Tirant lo Blanch, Valencia, 195-219.

McLACHLIN (2016): McLachlin, Beverley, "Accès à la justice et marginalisation: l'aspect humain de l'accès à la justice", en Les Cahiers de Droit 57/2, 341-350. Disponible en https://www.erudit.org/en/journals/cd1/2016-v57-n2cd02519/1036489ar.pdf

OEISM (2009): Observatorio sobre la Exclusión social y los procesos de Inclusión en la Comunidad de Madrid, De la exclusión a la ciudadanía. Análisis de los factores, barreras y oportunidades. Disponible en https://observatorioinclusion.files.wordpress.com/2013/07/informe-de-la-exclusiona-la-ciudadania.pdf

OEISM (2012): Observatorio sobre la Exclusión social y los procesos de Inclusión en la Comunidad de Madrid, "La promoción de la ciudadanía como clave de una intervención inclusiva y participativa", en Cuadernos de Trabajo social 25/2 (2012) 393-402. Disponible

en https://observatorioinclusion.files.wordpress.com/2012/04/promocion_ciudadania_ oeism.pdf

ONU (2015): https://www.un.org/sustainabledevelopment/es/ 
PATHFINDERS (2020): PATHFINDERS FOR PEACEFUL, JUST AND INCLUSIVE SOCIETIES, Justicia para todos, Disponible en https://bf889554$\underline{6857-4 c f e-8 d 55-}$ $\underline{\text { 8770007b8841.filesusr.com/ugd/90b3d6_1656bf93a96a4f35a3625f7c0e5a2f9d.pdf }}$

PLANCHADELL (2015): Planchadell Gargallo, Andrea: "La consecución de la tutela judicial efectiva en la litigación colectiva", en InDret. Revista para el análisis del Derecho 4/2015. Disponible en https://indret.com/wpcontent/themes/indret/pdf/1180_es.pdf

SANTOS (2018): Santos, Almudena, "El probono legal: Win-Win para ONGs, abogados y empresas". Disponible en https://elderecho.com/el-probono-legal-winwin-para-ongs-abogados-y-empresas

SAUQUILLO (2021): Sauquillo, Francisca (Coord.), Decálogo para la protección de los derechos humanos en pandemias, Fundación Abogacía española. Disponible en https://www.abogacia.es/wp-content/uploads/2021/02/Decalogo-Resolucion$\underline{\text { web.pdf }}$

UNESCO (2006), "Alfabetización funcional", en https://learningportal.iiep.unesco.org/en/node/7205

TRATADO (2012): Versión consolidada del Tratado de la Unión europea: https://eur-lex.europa.eu/resource.html?uri=cellar:2bf140bf-a3f8-4ab2-b506$\underline{\mathrm{fd} 71826 \mathrm{e} 6 \mathrm{da} 6.0005 .02 / \mathrm{DOC} \_1 \& \text { format=PDF }}$

WILCZYNSKI (2014): Wilczynski, Ania/Karras Maria/Forell Suzie, “The outcomes of community legal education: a systematic review", Justice Iusses 18. Disponible en http://www.lawfoundation.net.au/ljf/site/articleIDs/18C587ECBD959D50CA257A 91001F76F0/\$file/JI18_Outcomes_of_CLE_FINAL_web.pdf 
WORK (2018): Work for Social, Voluntariado profesional y pro bono. Diferencias, ventajas y buenas prácticas. Disponible en https://workforsocial.org/wpcontent/uploads/2018/07/Grupo-Pro-bono_25072018_ultima.pdf

WORLD (2019): World Justice Project, https://worldjusticeproject.org/our$\underline{\text { work/research-and-data/global-insights-access-justice-2019 }}$

WORLD (2020a): World Justice Project, https://worldjusticeproject.org/sites/default/files/documents/16.3.3\%20Flyer_Acces s\%20to\%20Civil\%20Justice_final_es.pdf

WORLD (2020b): World Project, https://worldjusticeproject.org/sites/default/files/documents/WJP-Global-ROLISpanish.pdf

WORLD (2020c), World Justice Project, https://worldjusticeproject.org/rule-oflaw-index/factors/2020/Spain/Open\%20Government/

Otra webgrafía:

http://www.ub.edu/experiencia/programes.php

http://www.ub.edu/grupapsub/es/article/nou-catàleg-compartir-idees-edició-2020

https://e-justice.europa.eu/content_legal_aid-37129-es.do

https://law.wisc.edu/law-in-action/

https://www.ine.es/dyngs/ODS/es/objetivo.htm?id=5252

https://www.lawinaction.ca/

https://www.thelegaleducationfoundation.org/ 